\title{
Non-Coding RNA as Biomarkers for Type 2 Diabetes Development and Clinical Management
}

\begin{abstract}
Tiange Chi ${ }^{1,2 \dagger}$, Jiaran $\mathrm{Lin}^{3,4 t}$, Mina Wang ${ }^{4,5 t}$, Yihan Zhao ${ }^{4}$, Zehuan Liao ${ }^{6,7 *}$ and Peng Wei ${ }^{1 *}$
1 School of Traditional Chinese Medicine, Beijing University of Chinese Medicine, Beijing, China, ${ }^{2}$ First Clinical Medical College, Beijing University of Chinese Medicine, Beijing, China, ${ }^{3}$ Department of Nephrology and Endocrinology, Dongzhimen Hospital Affiliated to Beijing University of Chinese Medicine, Beijing, China, ${ }^{4}$ Graduate School, Beijing University of Chinese Medicine, Beijing, China, ${ }^{5}$ Department of Acupuncture and Moxibustion, Beijing Hospital of Traditional Chinese Medicine, Capital Medical University, Beijing Key Laboratory of Acupuncture Neuromodulation, Beijing, China, ${ }^{6}$ School of Biological Sciences, Nanyang Technological University, Singapore, Singapore, ${ }^{7}$ Department of Microbiology, Tumor and Cell Biology (MTC), Karolinska Institutet, Stockholm, Sweden
\end{abstract}

OPEN ACCESS

Edited by:

Boon-How Chew,

Putra Malaysia University, Malaysia

Reviewed by:

Lihong Zhang,

Fuwai Hospital, China

Yan Xu,

Peking University, China

*Correspondence:

Peng Wei

weipeng@bucm.edu.cn

Zehuan Liao

liao0058@e.ntu.edu.sg

${ }^{\dagger}$ These authors have contributed equally to this work

Specialty section: This article was submitted to Clinical Diabetes,

a section of the journal

Frontiers in Endocrinology

Received: 16 November 2020

Accepted: 10 August 2021

Published: 17 September 2021

Citation:

Chi T, Lin J, Wang M, Zhao $Y$, Liao $Z$ and Wei $P$ (2021) Non-Coding

RNA as Biomarkers for Type 2

Diabetes Development and

Clinical Management.

Front. Endocrinol. 12:630032. doi: 10.3389/fendo.2021.630032
Diabetes, a metabolic disease characterized by high blood glucose and other complications, has undefined causes and multiple risk factors, including inappropriate diet, unhealthy lifestyles, and genetic predisposition. The two most distinguished types of diabetes are type 1 and type 2 diabetes, resulting from the autoimmune impairment of insulin-generating pancreatic $\beta$ cells and insulin insensitivity, respectively. Non-coding RNAs (ncRNAs), a cohort of RNAs with little transcriptional value, have been found to exert substantial importance in epigenetic and posttranscriptional modulation of gene expression such as messenger RNA (mRNA) silencing. This review mainly focuses on the pathology of type 2 diabetes (T2D) and ncRNAs as potential biomarkers in T2D development and clinical management. We consolidate the pathogenesis, diagnosis, and current treatments of T2D, and present the existing evidence on changes in multiple types of ncRNAs in response to various pathological changes and dysfunctions in different stages of T2D.

Keywords: ncRNAs, miRNAs, IncRNAs, circRNAs, diabetes, biomarker

\section{INTRODUCTION}

Diabetes is a kind of metabolic disease characterized by a high level of blood glucose with multiple complications, including macrovascular complications (cardiovascular disease) and microvascular complications (e.g., diabetic kidney disease, diabetic retinopathy, and neuropathy), and higher risks of developing several types of cancer, which subsequently could result in decreased life quality and even death (1-8). Type 1 and type 2 diabetes are the two most common types of this disease. Type 1 diabetes (T1D) presents an absolute insulin deficiency resulting from the autoimmune impairment of insulin-generating $\beta$ cells, while type 2 diabetes (T2D) displays a relative insulin deficiency due to metabolic dysfunction (9). According to the estimation of International Diabetes Federation, the prevalence of diabetes increases dramatically; by 2045, diabetes is projected to affect approximately 700 million people worldwide, up from the previous estimation of 463 million in 2019. Moreover, 
approximately one in every two persons living with diabetes is undiagnosed. In 2019, diabetes causes over 4 million deaths globally in the $20-79$ years age range $(10,11)$. To be diagnosed as diabetic, one's blood glucose should be equal to or above certain values. According to the classification and diagnosis of diabetes by the American Diabetes Association (12), the methods and criteria are as follows: fasting plasma glucose test [FPG $\geq 126 \mathrm{mg} / \mathrm{dl}(7.0 \mathrm{~mm} / \mathrm{L})]$ and fasting are defined as no caloric intake for the past $8 \mathrm{~h}$ at least; 2 -h oral glucose tolerance test [OGTT $\geq 200 \mathrm{mg} / \mathrm{dl}(11.1 \mathrm{mmol} / \mathrm{L})$ ] and the test should be conducted strictly as described by WHO, using a glucose load containing the equivalent of $75 \mathrm{~g}$ anhydrous glucose dissolved in water; glycated hemoglobin test $[\mathrm{A} 1 \mathrm{C} \geq 6.5 \%$ (48 $\mathrm{mmol} / \mathrm{mol})]$; or random plasma glucose $\geq 200 \mathrm{mg} / \mathrm{dl}(11.1 \mathrm{mmol} / \mathrm{L})$, coupled with classic symptoms of hyperglycemia or hyperglycemic crisis. In the absence of definitive hyperglucose, at least two of the abnormal test results are required for the diagnosis of diabetes, either from one same sample or two separate ones.

Non-coding RNA (ncRNA) refers to a type of RNA that is not involved in producing proteins but plays a key role in cellular function and development of different diseases. More than $90 \%$ of human genome RNAs consist of ncRNAs, and they can be divided into several types based on their size (13). The small ncRNAs such as microRNAs (miRNAs) and longer ncRNAs including long non-coding RNAs (lncRNAs) have been discovered that their up- or downregulation may regulate endothelial function in the vasculature, which is associated with the occurrence of diabetes (Table 1) (50). Moreover, the development of islet autoimmunity and dysfunction of $\beta$ cells may result from the deregulation of immune-cell-specific T1D loci-associated lncRNAs and islet-specific lncRNAs, which leads to T1D (51). In addition, lncRNAs are linked to poor glycemic control, insulin resistance, senescence, and proinflammation in patients with T2D (45). Thus, the specificity that ncRNAs exert significant functions in adjusting cellular pathways and the development of diseases affects their expression patterns, which is reflected in various body fluids, make them ideal as biomarkers for diabetes. This review aims to consolidate the pathogenesis, diagnosis, and treatments of T2D, and the role of ncRNAs as biomarkers in progress and management of T2D.

\section{TYPE 2 DIABETES}

\subsection{Current Pathogenesis}

According to the American Diabetes Association (ADA) (12), diabetes can be classified into the following general categories: type 1 diabetes; type 2 diabetes; gestational diabetes mellitus; specific types of diabetes due to other causes, e.g., monogenic diabetes syndromes (such as neonatal diabetes and maturityonset diabetes of the young) and diseases of the exocrine pancreas (such as cystic fibrosis and pancreatitis); and drug- or chemical-induced diabetes (such as with glucocorticoid use, in the treatment of HIV/AIDS, or after organ transplantation).

T2D is characterized by hyperglycemia, insulin resistance, and relatively impaired insulin secretion. Ever since the study of the
TABLE 1 | Summary of different expressions of ncRNAs in various target cells of T2D and prediabetic patients.

\begin{tabular}{|c|c|c|c|}
\hline & $\begin{array}{c}\text { Differentially Expressed } \\
\text { ncRNAs }\end{array}$ & Target Cells & Reference \\
\hline \multicolumn{4}{|l|}{ Enhanced $\uparrow$} \\
\hline \multirow[t]{5}{*}{ T2D Patients } & miR-16 & Pancreatic $\beta$ cells & (14) \\
\hline & CDR1 & Pancreatic $\beta$ cells & $(15,16)$ \\
\hline & circRNA-HIPK3 & Pancreatic $\beta$ cells & (16) \\
\hline & hsa_circ_0054633 & Pancreatic $\beta$ cells & (17) \\
\hline & circANKRD36 & Pancreatic $\beta$ cells & (18) \\
\hline \multirow{3}{*}{$\begin{array}{l}\text { Prediabetes } \\
\text { Patients }\end{array}$} & miR-499-5p & Hepatic cells & $(19,20)$ \\
\hline & & & \\
\hline & hsa_circ_0054633 & Pancreatic $\beta$ cells & (17) \\
\hline \multicolumn{4}{|l|}{ Reduced $\downarrow$} \\
\hline \multirow[t]{22}{*}{ T2D Patients } & miR-376 & Pancreatic $\beta$ cells & (21) \\
\hline & miR-432 & Pancreatic $\beta$ cells & (21) \\
\hline & miR-200 & Pancreatic $\beta$ cells & (22) \\
\hline & miR-184 & Pancreatic $\beta$ cells & (23) \\
\hline & miR-204 & Pancreatic $\beta$ cells & (24) \\
\hline & $\begin{array}{l}\text { miR-24, miR-26, miR-148, } \\
\text { miR-182 }\end{array}$ & Pancreatic $\beta$ cells & (25) \\
\hline & miR-9 & Pancreatic $\beta$ cells & (26) \\
\hline & $\begin{array}{l}\text { miR-130a, miR-130b, miR- } \\
152\end{array}$ & Pancreatic $\beta$ cells & (27) \\
\hline & miR-187 & Pancreatic $\beta$ cells & (28) \\
\hline & miR-7 & Pancreatic $\beta$ cells & (29) \\
\hline & miR-708 & Pancreatic $\beta$ cells & (30) \\
\hline & miR-34a, miR-146a & Pancreatic $\beta$ cells & $(31,32)$ \\
\hline & $\begin{array}{l}\text { miR-182-5p, miR-33, miR- } \\
37\end{array}$ & Pancreatic $\beta$ cells & $(33,34)$ \\
\hline & miR-802 & Hepatic cells & $(35,36)$ \\
\hline & miR-122-5p & Hepatic cells & $(37)$ \\
\hline & miR-106b & Skeletal muscle cells & $(38,39)$ \\
\hline & microRNA let-7a, let-7d & Skeletal muscle cells & $(40)$ \\
\hline & miR-29 & Skeletal muscle cells & (41) \\
\hline & $\begin{array}{l}\text { miR-192, miR-122, miR- } \\
\text { 27a-3p, miR-27b-3p }\end{array}$ & Adipocytes & (42) \\
\hline & LncRNA H19 & Pancreatic $\beta$ cells & $(43,44)$ \\
\hline & LncRNA MEG3 & Pancreatic $\beta$ cells & $(45-47)$ \\
\hline & LncRNA MALAT1 & Pancreatic $\beta$ cells & $(48,49)$ \\
\hline
\end{tabular}

impaired responsiveness to insulin of the diabetics opened by Berson and Yalow in the 1960s (52), insulin resistance has been proposed, investigated, demonstrated, and concluded as the initial defect in T2D. To compensate for the insufficient insulin functions due to the presence of insulin resistance, hyperinsulinemia occurs, consequent to increased $\beta$-cell insulin secretion. However, it is worth noting that patients with primary insulin resistance, characterized by marked hyperinsulinemia and genetically dysfunctional insulin receptor, namely, those with type A insulin resistance, Rabson-Mandenhall syndrome, or Leprechaunism, may have close to normal glucose tolerance, retain normal weight, and normotrygliceridemic, in spite of congenital significantly elevated plasma insulin concentrations (53). Therefore, it is the secondary insulin resistance that is being discussed here, which is remarkably associated with T2D. In a popular context of chronic energy surplus, usually caused by sedentary lifestyle, adipocyte dysfunction may arise as a result of fibro-inflammation process, when white adipose tissue fails to properly adapt and expand in response to positive energy balance, which is normally induced by insulin, liver, pancreas, and skeletal muscle, T2D occurs (54). 
It is widely assumed that aside from the aged tendency of population, changes in diet and lifestyle are also responsible for the speedy boost in the global prevalence and incidence of type 2 diabetes in recent decades. These two factors also contribute largely to the ongoing global obesity epidemic, while obesity is tightly correlated with the incidence of T2D (55). Epidemiological studies have shown that the level of overall physical exercise is related to a decline in the relative risk of diabetes by roughly $30 \%$ (56). There are also some evidence that T2D may derive from infection. For example, Chlamydia pneumoniae may induce $\beta$-cell dysfunction in the case of systemic inflammation (57). Although lifestyle and overeating seem to be the activating factors, genetic factors also play an important role in the pathogenesis of T2D. GWAS published in 2007 identified six new diabetes susceptibility genes: SLC30A8, HHEX-IDE, CDKN2A/2B, IGF2BP2, CDKAL1, and FTO (58-62). The first GWAS (61) repeated the previously known correlation between TCF7L2 and type 2 diabetes, which has been found in Icelandic populations (63). TCF7L2 is the most replicated genetic variant related to $\mathrm{T} 2 \mathrm{D}$ so far, with a relative risk of 1.4. Besides, epigenetic factors (such as DNA methylation) are particularly crucial as they may mediate the impact of environmental exposure to T2D (64).

\section{DIFFERENT TYPES OF NCRNAS USED AS BIOMARKERS OF DIABETES}

Non-coding RNA (ncRNA) is a functional RNA molecule that is not translated into a protein. The ncRNAs, making up more than
$90 \%$ of human genome RNAs, were once labeled "molecular fossils" or "relics" due to their conserved nature and for being "useless" transcriptional products. However, countless significant functions have been unveiled during the past decades, including recruitment of epigenetic modifier proteins, control of mRNA decay and translation, and DNA sequestration of transcription factors, showcasing tremendous biological and medical potential with fast-gaining momentum (65). Categorized by size and length, members of ncRNA family include miRNAs, siRNAs, piRNAs, snoRNAs, snRNAs, exRNAs, scaRNAs, and the long ncRNAs, and the nonlinear circular RNAs. Furthermore, ncRNAs such as miRNAs, IncRNAs, and circRNAs have been proven to have tight links or direct participation in the pathogenesis, development, and prognosis of T2D. Due to their unique roles in modulating biological actions preceding the changes on glucose level and improved detectability and accuracy boosted by technological progression, ncRNAs are emerging as potent biomarkers in the diagnosis of the development and clinical management, utilized alone or complementary to the traditional yardsticks (Figure 1).

\section{1 miRNA}

miRNAs are a large group of small (15-22 nts), non-coding sequences with hairpin conformation, and are highly conserved among the species. Their major roles include direct posttranscriptional repression or cleavage of mRNA targets, resulting in the destabilization of the transcripts, functioning as a critical regulator of an overarching array of cellular processes such as cell development, proliferation, differentiation, apoptosis, and metabolism (66). miRNAs have underestimated potentials,

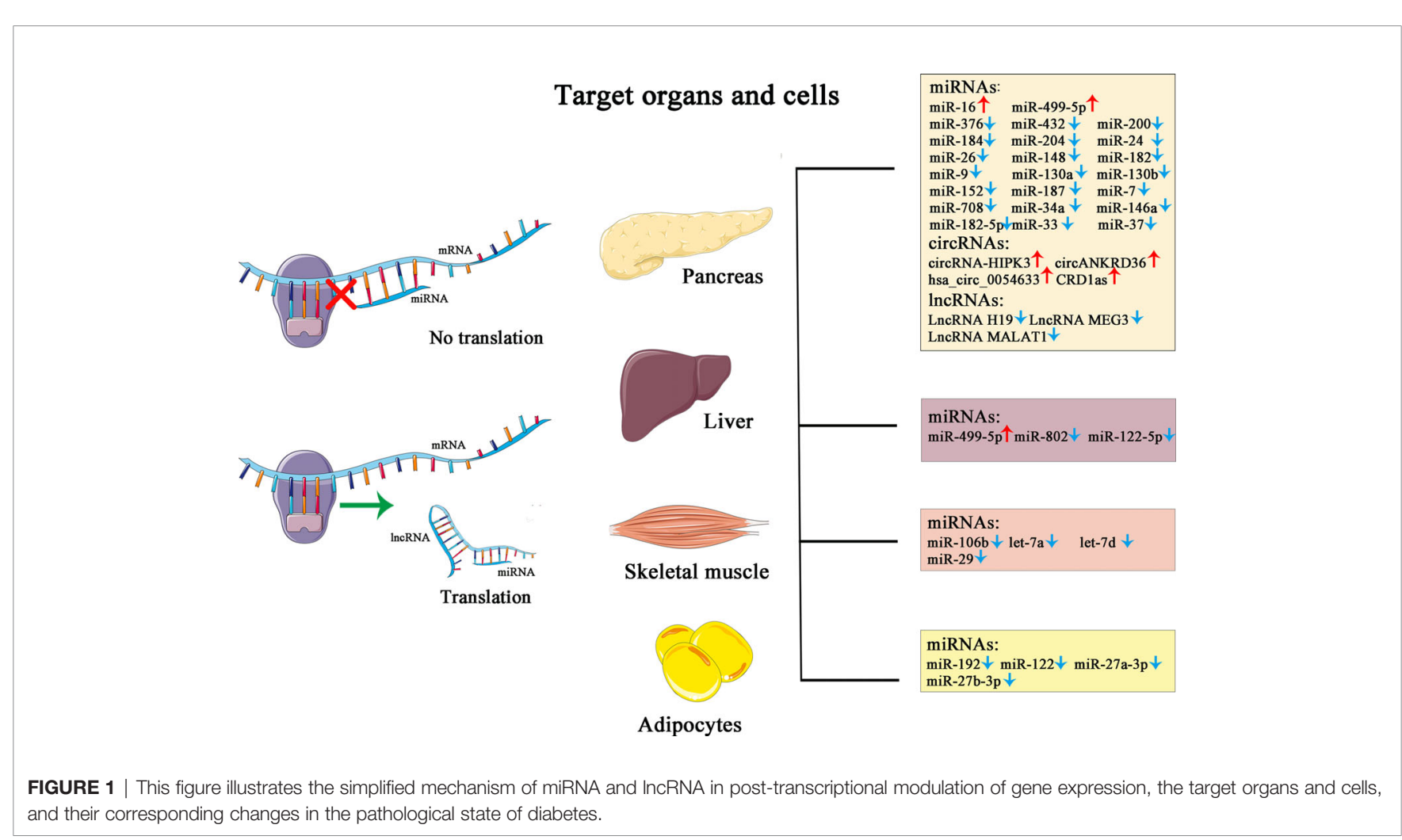


owing to technological limitations. It is only until the discovery of two miRNAs, lin-4 and let-7, which occupies pivotal niches in the timing of development of Caenorhabditis elegans. There are over 2,000 miRNAs listed for Homo sapiens, and it is predicted that approximately $60 \%$ of protein-coding human genes possess miRNA target sites $(67,68)$.

\subsection{1 miRNA and Pancreatic $\beta$ Cells}

The orchestra between pancreatic $\beta$ cells, insulin, and peripheral recipient cells including hepatic cells, skeletal muscle cells, and adipocytes is key to the occurrence of T2D. Therefore, apart from the conventional focus on the insulin insensitivity of recipient cells where blood glucose fails to enter or being stored as glycogen, attention should also be placed on the secretion and transportation of insulin as well. It has been proven that defective insulin secretion shares association with both reduced $\beta$-cell mass and impaired $\beta$-cell function, where miRNAs exert effect on (69).

The secretory functions of $\beta$ cell relies on its physical existence, with emphasis on the proliferation and apoptosis thereof. In human T2D islets, the incidence of methylation of the DLK1-MEG3 cluster is high. This region contains cellspecific histone modifications and is home to over 50 miRNAs, among whom miR-376 and miR-432 has been exemplified to control islet amyloid polypeptide (IAPP) level, which is involved in $\beta$-cell apoptosis (21). It has also been found that the expression of miR-200 is highly induced in the islets of diabetic mice, whose beta-cell-specific overexpression suffices to induce beta cell apoptosis and lethal T2D, while its ablation can rescue $\beta$ cells against apoptosis (22). The downregulation of miR-184 has been witnessed both in vitro and in vivo in obese diabetic mice, and a decreased expression in the islets of T2D patients, which corroborates the inhibitory effect of miRNA on beta-cell proliferation (23). Another phenomenon concerning beta-cell survival worth mentioning is the extracellular miR-16, which is delivered to $\beta$ cell by exosome-like vesicles released by skeletal muscles in insulin-resistant conditions. Probably as a compensatory effect, the upregulated expression of said miRNA is in favor of insulin-secreting cell expansion, which increases islet size (14).

Intact as the cell structure may be, pancreatic $\beta$ cell loses its existential significance once the secretory function proves useless. Before reaching target cells, insulin has to be synthesized and transported out of $\beta$ cells. In diabetic mice models and cultured beta cells, miR-204 expression has been demonstrated to be induced, which blocks insulin production by directly targeting and downregulating MAFA, a known insulin transcription factor (24). A similar effect has been witnessed in line with the downregulation of a cohort of miRNAs, namely, miR-24, miR-26, miR-148, and miR-182, in isolated islets and cultured $\beta$ cells where insulin content has been shown to be decreased due to reduced insulin promoter activity (25). MiR-9, among the first detected islet miRNAs, is one of the modulators of insulin granule exocytosis. Its increased expression represses the transcription factor ONECUT2 and increases the expression of granuphilin, which negatively regulates of insulin exocytosis
(26). Intracellular energy production also plays a role in the secretion of insulin granules. miR-130a, miR-130b, and miR-152 are found to have overexpression in T2D patient islets, where they each reduced the level of the common target pyruvate dehydrogenase E1 alpha 1 subunit (PDHA1), thereby reducing intracellular ATP and insulin secretion (27). According to a global profiling of islet miRNAs in cohorts of individuals with and without T2D conducted by Locke et al., a dramatic increase in the expression of miR-187 in the islets obtained from T2D donors was noticed. Its exact mechanism on insulin secretion remains to be precisely defined; however, certain relations with homeodomain-interacting protein kinase 3 (HIPK3) were suspected, a protein kinase that is required for normal insulin secretion and a direct target of this miRNA (28).

miR-7 is abundantly expressed in pancreatic islet cells. Studies showed that miR-7 directly regulates insulin granule exocytosis by controlling late stages of insulin granule fusion with the plasma membrane and ternary SNARE complex activity, with no effect on cell proliferation and apoptosis (29). Transgenic mice overexpressing miR-7a in $\beta$ cells developed diabetes due to impaired insulin secretion and $\beta$ cell dedifferentiation, while in human, its pattern of expression oscillates responding to the extent of insulin resistance: it is reduced under moderate insulin resistance conditions, contributing to improved insulin secretion; however, it raises progressively under severe diabetic conditions and can reach levels even higher than in healthy individuals (29). The abovementioned phenomenon has established itself as a major obstacle in applying the expression of miR-7 as an independent or complementary biomarker in determining the diagnosis of T2D. In addition to that, challenges for the clinical application of miRNAs as biomarkers also include but not limited to genetic background, treatment types, glycemic control quality, and disease duration.

Reactive oxygen species (ROS) has been placing a great threat to various tissues/cells, causing pathologies including inflammation, metabolic dysfunction, age-related degeneration, and diabetes. $\beta$ Cells may be at higher risk of oxidative damage from ROS, as a consequence of excessive levels of mitochondrial ROS generation and reduced nicotinamide adenine dinucleotide phosphate (NADPH) oxidase activity, failure of antioxidant defense, and endoplasmic reticulum (ER) stress (38). Chronic hyperglycemia and hyperlipidemia are characteristic of patients with T2D. Several studies have indicated that mammalian (human and murine) pancreatic islets cultured at high glucose concentration manifested miR-708 upregulation, treated with ER stress reliever, which was known to improve ER folding capacity; however, this upregulation is reversed (30). High levels of free fatty acids (FFAs) can be another trigger for beta-cell oxidative stress. Related miRNA such as miR34a and miR-146a showed an increasing pattern after palmitate treatment on islet cells, in parallel with increased beta-cell apoptotic behaviors, whose inhibition rescued the viability of beta-cells but not their insulin secretory functions $(31,32)$. Other miRNA with negative regulation on $\beta$-cell survival include miR-182-5p, miR-33, and miR-370, whose effect could be corrected by thrombospondin 1 (THBS-1) and Glucagon Like Peptide-1 (GLP-1), respectively $(33,34)$. 


\subsection{2 miRNA and Peripheral Recipient Cells}

Insulin resistance is the inability of the target tissues to orchestrate well-coordinated glucose-lowering processes, including the suppression of gluconeogenesis, lipolysis, net glycogen synthesis, and cellular glucose uptake in response to physiological blood insulin levels (70). The glucose-insulin balance is maintained through the liver, skeletal muscle, and white adipose tissue, with the liver exerting major effects as the metabolic center of an organism. Hence, once the hepatic insulin signaling cascade faces impairment resulting in hepatic insulin resistance, other metabolic symptoms occur, incurring hyperglycemia, inflammation, and de novo lipogenesis, and further, hepatic steatosis and nonalcoholic fatty liver disease (NAFLD) (67). Thus, it is beyond reasonable that tools for early diagnosis of hepatic insulin resistance is beneficial. miRNAs has been investigated for their potential as biomarkers for hepatic insulin resistance, and to qualify as one, whose circulating levels must correlate to corresponding hepatic states and must be crucial in the signaling cascade. miR-802, an intensively studied miRNA in the oncological field, has been demonstrated to be associated with oxidative stress and hepatic insulin resistance. In high-fat diet (HFD) mice, studies have shown that, along with increased expression of miR-802, ROS generation was significantly greater, and the expression of gluconeogenesis-related genes was significantly downregulated $(35,36)$, and its circulating levels were dramatically elevated in T2D patients, qualifying it as a biomarker. Other miRNAs with changed expression patterns include miR-499-5p, which affects insulin signaling cascade and glycogen synthesis by suppressing phosphatase and tensin homolog (PTEN), alongside the improvement of Akt/GSK activation, and is found to be reduced in prediabetic patients $(19,20)$, and miR-122-5p, which affects the hepatic gluconeogenesis process, whose circulating levels were significantly higher in cohorts with insulin resistance, T2D, or MetS (37).

Aside from liver cells, another important target of insulin is skeletal muscle, whose glucose uptake also substantially contributes to glucose and metabolic homeostasis. In an investigation on the antioxidant effects of berberine (BBR), an isoquinoline alkaloid, it was found that BBR could attenuate oxidative stress of diabetic mice partly through inhibiting miR106b/SIRT1 pathway, an miRNA associated with skeletal muscle insulin resistance $(38,39)$. Interleukin (IL)-13 has been revealed having an autocrine role in the glucose metabolism of skeletal muscle according to Jiang et al., and its exposure increases skeletal muscle glucose uptake, oxidation, and glycogen synthesis via an Akt-dependent mechanism. In T2D patients, such bioactivities are found to be suppressed by the increased expression of miRNA let-7a and let-7d, which repress IL-13 genes on translational level (40). To put it simpler, insulin functions by combining to insulin receptor substrate 1 (IRS-1), subsequently triggering a signaling cascade consisting of phosphorylation of protein kinase $\mathrm{B}(\mathrm{PKB} / \mathrm{AKT})$, translocation of glucose transporter-4 (Glut4) from the cytosol to the membrane, and glucose uptake. The overexpression of miR-29 was demonstrated to be capable of disrupting the glucose metabolism of skeletal muscle by inhibiting insulin signaling, expression of insulin receptor substrate 1 (IRS-1), and phosphoinositide 3-kinase (41).

Since obesity are getting more and more attention as a risk factor of $\mathrm{T} 2 \mathrm{D}$, the role white adipose tissues play in diabetes is receiving piling investigational highlights. Researchers have found that certain exosomes extracted from obese mice were able to induce glucose intolerance in lean mice transfected with certain exosomes, which was demonstrated to be viable through the miRNA content, namely, miR-192, miR-122, miR-27a-3p, and miR-27b-3p, the expression of all of which was increased in obese mice. Data further showed that the mechanism of induced diabetes in white adipose tissue was navigated by the targeting of peroxisome proliferator-activated receptor $\alpha(\operatorname{PPAR} \alpha)(42,71)$.

\subsection{IncRNA}

lncRNAs are a group of transcripts, with lengths extending 200 nucleotides, that also do not translate into proteins. Divided by functions, lncRNAs have three subtypes: the non-functional ones, which serve no purpose other than being by-products of transcriptions; the second type are those whose own transcription is of a self-sufficient manner; and the third type consists of those that are able to act in cis and/or trans orientations (72). IncRNAs interact intensively with miRNAs, acting as the molecular sponges or decoys of miRNAs to regulate their cytoplasmic level by binding specific miRNAs and actively sequestrate them from their target mRNAs. In turn, lncRNAs can be destabilized by miRNAs' direct targeting and posed as competitors on shared mRNA targets (67). With such intertwined involvement in gene expression regulation, lncRNAs have been presenting some potential in T2D development and management.

H19 is among the earliest-discovered lncRNAs, whose biofunctions have been relatively thoroughly investigated. A recent study conducted by Sanchez-Parra et al. found that H19 may act upstream of miRNA let-7 and the activation of Akt, whose silencing decreased $\beta$-cell expansion in newborns and reexpression promoted proliferation of $\beta$ cells in adults (43). Moreover, the circulating levels of $\mathrm{H} 19$ have been demonstrated to be significantly increased in T2D patient cohorts, according to Fawzy et al., which indicates its potential as a biomarker in insulin resistance (44).

MEG3 is another lncRNA known to researchers for a long time as well, mostly indicated in cancer suppression. Its association has been noted with pancreatic cell apoptosis, insulin synthesis, and secretion, whose expression may function as a new regulator of maintaining beta cells identity (46). However, its overexpression, suggested by Zhu et al., may be a promoter for hepatic insulin resistance via increasing FOXO1 expression by acting as a sponge for miR-214 (47). It has also been reported that the competitive binding of MEG3 to miR-185-5p, acting as a competing endogenous RNA (ceRNA), promoted the expression of early growth response 2 (EGR2), which was reported to inhibit IRS. Clinical evidence also supported this hypothesis: its overexpression in T2D patients was significant (45).

Like its counterparts, IncRNAs are associated with oxidative stress-induced insulin resistance as well, according to studies. 
lncRNA MALAT1, which has been shown to take part in regulation of cell proliferation and motility, has been found to partake in suppressing insulin signaling by inhibiting the phosphorylation of IRS and Akt, via the upregulation of the c-Jun N-terminal kinase (Jnk), a stress-sensitive kinase (48). This negative regulatory effect has been corroborated by its increased expression in GDM patients (49).

\section{3 circRNAs}

Circular RNAs (circRNAs) are a group of non-linear, naturally occurring ncRNAs with covalently closed circular structures. They are usually generated from precursor mRNA by a noncanonical event called backsplicing, displaying exceptional stability and evolutionary conservation, and tissue or development stage-specific expression patterns (73). Compared to miRNAs and lncRNAs, where many research results have been obtained, the biological functions discovered for circRNAs are relatively scarce, whereas a few associations have been made between them and glucose metabolism, insulin resistance, and T2D.

Certain circRNAs are capable of fulfilling various intracellular functions mainly by acting as sponges of miRNAs or RNAbinding proteins (RBPs). The best understood endogenous circRNA to this date has been CDR1as (also termed as ciRS-7). By potently binding to miR-7, it relieves the inhibitory effect of miR-7 on $\beta$-cell function, subsequently promoting islet $\beta$-cells proliferation and insulin secretion (15). Such phenomenon has also been observed in vivo, where the expression of CDRlas was reduced in diabetic mice (16). A similar effect has been witnessed in another circRNA, circRNA-HIPK3, whose regulation on islet cell function was mediated by sequestering miR-124-3p and miR-338-3p (16). Clinically, such effect could be potentially employed as a marker for therapeutic outcome evaluation. Reverse transcription polymerase chain reaction (RT-PCR) and quantitative polymerase chain reaction (qPCR) analyses were adopted for the screening of circRNAs related to T2D, and several circRNAs were detected to show statistical significance between T2D patients and healthy controls. Among them, there are hsa_circ_0054633 and circANKRD36, with the former being a potential diagnostic biomarker of prediabetes and T2D in peripheral blood cells and the latter displaying a potential in discerning the T2D-inflicted within cohorts with chronic inflammation $(17,18)$.

\section{DISCUSSION}

Early detection of diabetes mellitus is very useful for preventing onset and progression. Several biomarkers of diabetes mellitus have been reported, such as GDF-15, YKL-40, 2-aminoadipic acid, serum adipocyte fatty acid-binding protein, and urinary 8-oxo-7,8-dihydro-2'-deoxyguanosine (74-78). While many current biomarkers are based on proteins, ncRNAs have been recognized as a new sensitive, noninvasive biomarker for diagnosis, prognosis, and prediction to therapeutic responses in the recent years due to their high stability in body fluids (urine, plasma, exosomes, etc.) and the development of new detection techniques (79).

miRNAs are very stable and resistant to ribonucleases, freezing/thawing cycles, and other severe experimental conditions (80). Therefore, serum or plasma samples can be stored at $-20^{\circ} \mathrm{C}$ or $-80^{\circ} \mathrm{C}$ for several months without causing significant degradation of miRNAs, which serves as valid evidence to support the use of miRNAs as ideal biomarkers (81). lncRNAs are less stable than miRNAs, but compared to protein-coding mRNAs and miRNAs that are frequently expressed in multiple tissues, they show higher tissue specificity (82) and tend to show remarkable level of overexpression in diabetes. circRNAs are presumably more stable than most linear RNAs because they form a unique, circular, covalently closed continuous loop that is resistant to exonuclease-mediated degradation as they have no $5^{\prime}$ or $3^{\prime}$ ends. Recent evidence indicates that circRNAs usually regulate the transcription of miRNA-target genes by acting as miRNA sponges.

The extensive role of ncRNAs in physiological processes and deregulation in human diseases also makes them very attractive targets for new therapies. Several strategies that either silence overexpressed ncRNAs or reactivate downregulated ncRNAs are currently being investigated. Some of the ncRNAs have even shown encouraging therapeutic effects in animal models. For instance, MEG3 knockdown in STZ-induced diabetic mice resulted in increased levels of acellular capillary formation, microvascular leakage, and inflammatory proteins (83). MIAT knockdown in STZ-induced diabetic rats did not affect body weight or blood glucose levels but corresponded with improvements in visual function and partial reversal of a-wave, b-wave, and oscillatory potentials. MIAT downregulation also decreased the number of apoptotic retinal cells and attenuated retinal vessel impairment and retinal vascular leakage (84). In short, the therapeutic potential of ncRNA has been discovered, but the current findings are only the tip of the iceberg, and further research is still needed.

However, the pivotal issue is how to apply the molecular markers determined in the laboratory to the clinical environment. Currently, various limitations impede this research field and delay the clinical application of ncRNAs. On the grounds that ncRNAs are a quite novel area of research, most of the information obtained from the previous studies is only descriptive and correlational, and it is not possible to precisely infer the cause and effect. In particular, the deficiency of thorough understanding regarding the explicit origin, synthesis, modification, and regulatory pathways, and the interactions, cross-talk and coregulation of ncRNAs may hinder the clinical utilization of these ncRNAs. Hence, more edge leading and precise approaches in ncRNAs expression quantification and functional analysis are required.

According to association studies, prevalent assertion arises that ncRNAs can be used as biomarkers for diabetes. Nonetheless, as mentioned above, correlation does not imply causation; thus, pilot results still require large-scale clinical trials. Furthermore, it is strongly advocated to perform in vitro/in vivo verification of ncRNAs with candidate predictive functions and therapeutic targets since what occurs under physiological and pathophysiological conditions may 
not necessarily be consistent with the results from pure calculation and analysis. Therefore, the current data should be regarded as exploratory data and illustrated discreetly before further experiments and comprehensive clinical verification can prove the biological effects of ncRNAs.

The tissue specificity of ncRNAs and their enhanced stability in body fluid also enable them to be a promising future antidiabetic therapy. However, ncRNA therapy that can be authentically applied in the clinics still faces considerable obstacles, including the development of reliable delivery systems, dosage regimens, and technologies to improve off-target effects. On top of these, just like any other therapies, it is expected that ncRNAs as drugs may cause adverse side effects or induce drug resistance.

\section{CONCLUSION}

ncRNAs have a role in regulation of cellular functions including development, proliferation, differentiation, and apoptosis. ncRNAs anomalies would be indicative as molecular signatures under disease states, which can be applied to arrive at different diagnoses and explore treatments. Considering the rawness of this research field, many ncRNAs functions are waiting to be discovered. In conclusion, studies combining ncRNAs and

\section{REFERENCES}

1. Tan Y, Wang M, Yang K, Chi T, Liao Z, Wei P. PPAR- $\alpha$ Modulators as Current and Potential Cancer Treatments. Front Oncol (2021) 11:707. doi: 10.3389/fonc. 2021.599995

2. Wang M, Xie F, Lin J, Zhao Y, Zhang Q, Liao Z, et al. Diagnostic and Prognostic Value of Circulating CircRNAs in Cancer. Front Med (2021) 8:231. doi: 10.3389/fmed.2021.649383

3. Yang Y, Yang T, Liu S, Cao Z, Zhao Y, Su X, et al. Concentrated Ambient PM2. 5 Exposure Affects Mice Sperm Quality and Testosterone Biosynthesis. PeerJ (2019) 7:e8109. doi: 10.7717/peerj.8109

4. Liao Z, Wong S, Yeo H, Zhao Y. Smart Nanocarriers for Cancer Treatment: Clinical Impact and Safety. NanoImpact (2020) p:100253. doi: 10.1016/ j.impact.2020.100253

5. Liao Z, Chua D, Tan NS. Reactive Oxygen Species: A Volatile Driver of Field Cancerization and Metastasis. Mol Cancer (2019) 18(1):1-10. doi: 10.1186/ s12943-019-0961-y

6. Liao Z, Tan Z, Zhu P, Tan N. Cancer-Associated Fibroblasts in Tumor Microenvironment-Accomplices in Tumor Malignancy. Cell Immunol (2019) 343:103729. doi: 10.1016/j.cellimm.2017.12.003

7. Cole JB, Florez JC. Genetics of Diabetes Mellitus and Diabetes Complications. Nat Rev Nephrol (2020) 16(7):377-90. doi: 10.1038/s41581-020-0278-5

8. Khan RMM, Chua ZJY, Tan JC, Yang Y, Liao Z, Zhao Y. From Pre-Diabetes to Diabetes: Diagnosis, Treatments and Translational Research. Medicina (2019) 55(9):546. doi: 10.3390/medicina55090546

9. Wang M, Yang Y, Liao Z. Diabetes and Cancer: Epidemiological and Biological Links. World J Diabetes (2020) 11(6):227-38. doi: 10.4239/ wjd.v11.i6.227

10. Williams R, Karuranga S, Malanda B, Saeedi P, Basit A, Besancon S, et al. IDF Atlas 9th Edition 2019. International Diabetes Federation. (2019).

11. Cho NH, Shaw JE, Karuranga S, Huang Y, da Rocha Fernande JD, Ohlrogge AW, et al. IDF Diabetes Atlas: Global Estimates of Diabetes Prevalence for 2017 and Projections for 2045. Diabetes Res Clin Pract (2018) 138:271-81. doi: 10.1016/j.diabres.2018.02.023

12. American Diabetes, A. 2. Classification and Diagnosis of Diabetes: Standards of Medical Care in Diabetes-2020. Diabetes Care (2020) 43(Suppl 1):S14-31. doi: $10.2337 / \mathrm{dc} 20-\mathrm{S} 002$ clinical, genetic, epigenetic, and classical markers could help direct the medical decisions on diabetic patients and show broad prospect in precision medicine.

\section{AUTHOR CONTRIBUTIONS}

Conceptualization: ZL and PW. Writing-original draft preparation: TC, JL, MW, and YZ. Writing-review and editing: ZL and PW. Supervision: ZL and PW. Funding acquisition: PW. All authors contributed to the article and approved the submitted version.

\section{FUNDING}

This work was funded by the National Natural Science Foundation of China (Grant No. 31800652), Young Scientist Program by Beijing University of Chinese Medicine (Grant No. BUCM-2019-QNKXJ-C014), and the "Double First Class" Construction Funds of Discipline of Integrated Traditional Chinese and Western Medicine of Beijing University of Chinese Medicine.

13. Slack FJ, Chinnaiyan AM. The Role of Non-Coding RNAs in Oncology. Cell (2019) 179(5):1033-55. doi: 10.1016/j.cell.2019.10.017

14. Nesca V, Guay C, Jacovetti C, Menoud V, Peyot ML, Laybutt DR, et al. Identification of Particular Groups of microRNAs That Positively or Negatively Impact on Beta Cell Function in Obese Models of Type 2 Diabetes. Diabetologia (2013) 56(10):2203-12. doi: 10.1007/s00125-013-2993-y

15. Memczak S, Jens M, Elefsinioti A, Torti F, Krueger J, Rybak A, et al. Circular RNAs Are a Large Class of Animal RNAs With Regulatory Potency. Nature (2013) 495(7441):333-8. doi: 10.1038/nature11928

16. Stoll L, Sobel J, Rodriguez-Trejo A, Guay C, Lee K, Venø MT, et al. Circular RNAs as Novel Regulators of Beta-Cell Functions in Normal and Disease Conditions. Mol Metab (2018) 9:69-83. doi: 10.1016/j.molmet.2018.01.010

17. Fang Y, Wang X, Li W, Han J, Jin J, Su F, et al. Screening of Circular RNAs and Validation of Circankrd36 Associated With Inflammation in Patients With Type 2 Diabetes Mellitus. Int J Mol Med (2018) 42(4):1865-74. doi: 10.3892/ ijmm.2018.3783

18. Zhao Z, Li X, Jian D, Hao P, Rao L, Li M. Hsa_circ_0054633 in Peripheral Blood can be Used as a Diagnostic Biomarker of Pre-Diabetes and Type 2 Diabetes Mellitus. Acta Diabetol (2017) 54(3):237-45. doi: 10.1007/s00592016-0943-0

19. Fluitt MB, Kumari N, Nunlee-Bland G, Nekhai S, Gambhir KK. miRNA-15a, miRNA-15b, and miRNA-499 Are Reduced in Erythrocytes of Pre-Diabetic African-American Adults. Jacobs J Diabetes Endocrinol (2016) 2(1):14.

20. Wang L, Zhang N, Pan HP, Wang Z, Cao ZY. MiR-499-5p Contributes to Hepatic Insulin Resistance by Suppressing PTEN. Cell Physiol Biochem (2015) 36(6):2357-65. doi: 10.1159/000430198

21. Hall E, Volkov P, Dayeh T, Esguerra JL, Salö S, Eliasson L, et al. Sex Differences in the Genome-Wide DNA Methylation Pattern and Impact on Gene Expression, microRNA Levels and Insulin Secretion in Human Pancreatic Islets. Genome Biol (2014) 15(12):522. doi: 10.1186/s13059-0140522-z

22. Belgardt B-F, Ahmed K, Spranger M, Latreille M, Denzler R, Kondratiuk N. The microRNA-200 Family Regulates Pancreatic Beta Cell Survival in Type 2 Diabetes. Nat Med (2015) 21(6):619-27. doi: 10.1038/nm.3862

23. Martinez-Sanchez A, Nguyen-Tu MS, Cebola I, Yavari A, Marchetti P, Piemonti L, et al. MiR-184 Expression Is Regulated by AMPK in Pancreatic Islets. FASEB J (2018) 32(5):2587-600. doi: 10.1096/fj.201701100R 
24. Xu G, Chen J, Jing G, Shalev A. Thioredoxin-Interacting Protein Regulates Insulin Transcription Through microRNA-204. Nat Med (2013) 19(9):11416. doi: $10.1038 / \mathrm{nm} .3287$

25. Melkman-Zehavi T, Oren R, Kredo-Russo S, Shapira T, Mandelbaum AD, Rivkin N. miRNAs Control Insulin Content in Pancreatic $\beta$-Cells via Downregulation of Transcriptional Repressors. EMBO J (2011) 30(5):83545. doi: 10.1038/emboj.2010.361

26. Plaisance V, Abderrahmani A, Perret-Menoud V, Jacquemin P, Lemaigre F, Regazzi R. MicroRNA-9 Controls the Expression of Granuphilin/Slp4 and the Secretory Response of Insulin-Producing Cells. J Biol Chem (2006) 281 (37):26932-42. doi: 10.1074/jbc.M601225200

27. Ofori JK, Salunkhe VA, Bagge A, Vishnu N, Nagao M, Mulder H, et al. Elevated miR-130a/Mir130b/miR-152 Expression Reduces Intracellular ATP Levels in the Pancreatic Beta Cell. Sci Rep (2017) 7:44986. doi: 10.1038/ srep44986

28. Locke JM, da Silva Xavier G, Dawe HR, Rutter GA, Harries LW. Increased Expression of miR-187 in Human Islets From Individuals With Type 2 Diabetes Is Associated With Reduced Glucose-Stimulated Insulin Secretion. Diabetologia (2014) 57(1):122-8. doi: 10.1007/s00125-013-3089-4

29. Latreille M, Hausser J, Stützer I, Zhang Q, Hastoy B, Gargani S, et al. MicroRNA-7a Regulates Pancreatic Beta Cell Function. J Clin Invest (2014) 124(6):2722-35. doi: 10.1172/JCI73066

30. Ozcan U, Yilmaz E, Ozcan L, Furuhashi M, Vaillancourt E, Smith RO, et al. Chemical Chaperones Reduce ER Stress and Restore Glucose Homeostasis in a Mouse Model of Type 2 Diabetes. Science (2006) 313(5790):1137-40. doi: $10.1126 /$ science.1128294

31. Lovis P, Roggli E, Laybutt DR, Gattesco S, Yang JY, Widmann C, et al. Alterations in microRNA Expression Contribute to Fatty Acid-Induced Pancreatic Beta-Cell Dysfunction. Diabetes (2008) 57(10):2728-36. doi: $10.2337 / \mathrm{db} 07-1252$

32. Saeedi Borujeni MJ, Esfandiary E, Baradaran A, Valiani A, Ghanadian M, Codoñer-Franch P, et al. Molecular Aspects of Pancreatic Beta-Cell Dysfunction: Oxidative Stress, microRNA, and Long Noncoding RNA. J Cell Physiol (2019) 234(6):8411-25. doi: 10.1002/jcp.27755

33. Zhang Z, Liu H, Li Q. Glucagon-Like Peptide-1 Effects Lipotoxic Oxidative Stress by Regulating the Expression of microRNAs. Biochem Biophys Res Commun (2017) 482(4):1462-8. doi: 10.1016/j.bbrc.2016.12.058

34. Liu Y, Dong J, Ren B. MicroRNA-182-5p Contributes to the Protective Effects of Thrombospondin 1 Against Lipotoxicity in INS-1 Cells. Exp Ther Med (2018) 16(6):5272-9. doi: 10.3892/etm.2018.6883

35. Yang X, Xing H, Liu J, Yang L, Ma H, Ma H. MicroRNA802 Increases Hepatic Oxidative Stress and Induces Insulin Resistance in Highfat Fed Mice. Mol Med Rep (2019) 20(2):1230-40. doi: 10.3892/mmr.2019.10347

36. Higuchi C, Nakatsuka A, Eguchi J, Teshigawara S, Kanzaki M, Katayama A, et al. Identification of Circulating miR-101, miR-375 and miR-802 as Biomarkers for Type 2 Diabetes. Metabolism (2015) 64(4):489-97. doi: 10.1016/j.metabol.2014.12.003

37. Willeit $\mathrm{P}$, Skroblin $\mathrm{P}$, Moschen AR, Yin X, Kaudewitz D, Zampetaki A, et al. Circulating MicroRNA-122 Is Associated With the Risk of New-Onset Metabolic Syndrome and Type 2 Diabetes. Diabetes (2017) 66(2):347-57. doi: $10.2337 / \mathrm{db} 16-0731$

38. Grieco GE, Brusco N, Licata G, Nigi L, Formichi C, Dotta F, et al. Targeting microRNAs as a Therapeutic Strategy to Reduce Oxidative Stress in Diabetes. Int J Mol Sci (2019) 20(24). doi: 10.3390/ijms20246358

39. Chen DL, Yang KY. Berberine Alleviates Oxidative Stress in Islets of Diabetic Mice by Inhibiting miR-106b Expression and Up-Regulating Sirt1. J Cell Biochem (2017) 118(12):4349-57. doi: 10.1002/jcb.26089

40. Jiang LQ, Franck N, Egan B, Sjögren RJ, Katayama M, Duque-Guimaraes D, et al. Autocrine Role of Interleukin-13 on Skeletal Muscle Glucose Metabolism in Type 2 Diabetic Patients Involves microRNA Let-7. Am J Physiol Endocrinol Metab (2013) 305(11):E1359-66. doi: 10.1152/ajpendo.00236.2013

41. Massart J, Sjögren RJO, Lundell LS, Mudry JM, Franck N, O'Gorman DJ, et al. Altered miR-29 Expression in Type 2 Diabetes Influences Glucose and Lipid Metabolism in Skeletal Muscle. Diabetes (2017) 66(7):1807-18. doi: 10.2337/ $\mathrm{db} 17-0141$

42. Castano C, Kalko S, Novials A, Párrizas M. Obesity-Associated Exosomal miRNAs Modulate Glucose and Lipid Metabolism in Mice. Proc Natl Acad Sci USA (2018) 115(48):12158-63. doi: 10.1073/pnas.1808855115
43. Sanchez-Parra C, Jacovetti C, Dumortier O, Lee K, Peyot ML, Guay C, et al. Contribution of the Long Noncoding RNA H19 to $\beta$-Cell Mass Expansion in Neonatal and Adult Rodents. Diabetes (2018) 67(11):2254. doi: 10.2337/db180201

44. Fawzy MS, Abdelghany AA, Toraih EA, Mohamed AM. Circulating Long Noncoding RNAs H19 and GAS5 Are Associated With Type 2 Diabetes But Not With Diabetic Retinopathy: A Preliminary Study. Bosn J Basic Med Sci (2020) 20(3):365-71. doi: 10.17305/bjbms.2019.4533

45. Sathishkumar C, Prabu P, Mohan V, Balasubramanyam M. Linking a Role of IncRNAs (Long Non-Coding RNAs) With Insulin Resistance, Accelerated Senescence, and Inflammation in Patients With Type 2 Diabetes. Hum Genomics (2018) 12(1):41. doi: 10.1186/s40246-018-0173-3

46. You L, Wang N, Yin D, Wang L, Jin F, Zhu Y, et al. Downregulation of Long Noncoding RNA Meg3 Affects Insulin Synthesis and Secretion in Mouse Pancreatic Beta Cells. J Cell Physiol (2016) 231(4):852-62. doi: 10.1002/ jcp. 25175

47. Zhu X, Wu YB, Zhou J, Kang DM. Upregulation of IncRNA MEG3 Promotes Hepatic Insulin Resistance via Increasing FoxO1 Expression. Biochem Biophys Res Commun (2016) 469(2):319-25. doi: 10.1016/j.bbrc.2015.11.048

48. Chen J, Ke S, Zhong L, Wu J, Tseng A, Morpurgo B, et al. Long Noncoding RNA MALAT1 Regulates Generation of Reactive Oxygen Species and the Insulin Responses in Male Mice. Biochem Pharmacol (2018) 152:94-103. doi: 10.1016/j.bcp.2018.03.019

49. Zhang $\mathrm{Y}, \mathrm{Wu} \mathrm{H}$, Wang $\mathrm{F}, \mathrm{Ye} \mathrm{M}, \mathrm{Zhu} \mathrm{H}, \mathrm{Bu}$ S. Long Non-Coding RNA MALAT1 Expression in Patients With Gestational Diabetes Mellitus. Int J Gynaecol Obstet (2018) 140(2):164-9. doi: 10.1002/ijgo.12384

50. Zhang HN, Xu QQ, Thakur A, Alfred MO, Chakraborty M, Ghosh A, et al. Endothelial Dysfunction in Diabetes and Hypertension: Role of microRNAs and Long Non-Coding RNAs. Life Sci (2018) 213:258-68. doi: 10.1016/ j.lfs. 2018.10 .028

51. Mirza AH, Kaur S, Pociot F. Long Non-Coding RNAs as Novel Players in $\beta$ Cell Function and Type 1 Diabetes. Hum Genomics (2017) 11(1):17. doi: 10.1186/s40246-017-0113-7

52. Yalow RS, Berson SA. Plasma Insulin Concentrations in Nondiabetic and Early Diabetic Subjects. Determinations by a New Sensitive Immuno-Assay Technic. Diabetes (1960) 9:254-60. doi: 10.2337/diab.9.4.254

53. Musso C, Cochran E, Moran SA, Skarulis MC, Oral EA, Taylor S, et al. Clinical Course of Genetic Diseases of the Insulin Receptor (Type A and Rabson-Mendenhall Syndromes): A 30-Year Prospective. Med (Baltimore) (2004) 83(4):209-22. doi: 10.1097/01.md.0000133625.73570.54

54. Sbraccia P, D’Adamo M, Guglielmi V. Is Type 2 Diabetes an Adiposity-Based Metabolic Disease? From the Origin of Insulin Resistance to the Concept of Dysfunctional Adipose Tissue. Eat Weight Disord (2021). doi: 10.1007/ s40519-021-01109-4

55. Ng M, Fleming T, Robinson M, Thomson B, Graetz N, Margono C, et al. Global, Regional, and National Prevalence of Overweight and Obesity in Children and Adults During 1980-2013: A Systematic Analysis for the Global Burden of Disease Study 2013. Lancet (2014) 384(9945):766-81. doi: 10.1016/ S0140-6736(14)60460-8

56. Aune D, Norat T, Leitzmann M, Tonstad S, Vatten LJ. Physical Activity and the Risk of Type 2 Diabetes: A Systematic Review and Dose-Response Meta-Analysis. Eur J Epidemiol (2015) 30(7):529-42. doi: 10.1007/s10654-015-0056-Z

57. Rodriguez AR, Plascencia-Villa G, Witt CM, Yu JJ, José-Yacamán M, Chambers JP, et al. Chlamydia Pneumoniae Promotes Dysfunction of Pancreatic Beta Cells. Cell Immunol (2015) 295(2):83-91. doi: 10.1016/ j.cellimm.2015.03.010

58. Zeggini E, Weedon MN, Lindgren CM, Frayling TM, Elliott KS, Lango H, et al. Replication of Genome-Wide Association Signals in UK Samples Reveals Risk Loci for Type 2 Diabetes. Science (2007) 316(5829):1336-41. doi: $10.1126 /$ science.1142364

59. Saxena R, Voight BF, Valeriya L, Burtt NP, De Bakker PIW, Chen H, et al. Genome-Wide Association Analysis Identifies Loci for Type 2 Diabetes and Triglyceride Levels. Science (2007) 316(5829):1331-6. doi: 10.1126/ science. 1142358

60. Scott LJ, Mohlke KL, Bonnycastle LL, Willer CJ, Li Y, Duren WL, et al. A Genome-Wide Association Study of Type 2 Diabetes in Finns Detects Multiple Susceptibility Variants. Science (2007) 316(5829):1341-5. doi: $10.1126 /$ science. 1142382 
61. Sladek R, Rocheleau G, Rung J, Dina C, Shen L, Serre D, et al. A GenomeWide Association Study Identifies Novel Risk Loci for Type 2 Diabetes. Nature (2007) 445(7130):881-5. doi: 10.1038/nature05616

62. Steinthorsdottir V, Thorleifsson G, Reynisdottir I, Benediktsson R, Jonsdottir T, Walters GB, et al. A Variant in CDKAL1 Influences Insulin Response and Risk of Type 2 Diabetes. Nat Genet (2007) 39(6):770-5. doi: 10.1038/ng2043

63. Grant SF, Thorleifsson G, Reynisdottir I, Benediktsson R, Manolescu A, Sainz J, et al. Variant of Transcription Factor 7-Like 2 (TCF7L2) Gene Confers Risk of Type 2 Diabetes. Nat Genet (2006) 38(3):320-3. doi: 10.1038/ng1732

64. Davegårdh C, García-Calzón S, Bacos K, Ling C. DNA Methylation in the Pathogenesis of Type 2 Diabetes in Humans. Mol Metab (2018) 14:12-25. doi: 10.1016/j.molmet.2018.01.022

65. Chang W, Wang J. Exosomes and Their Noncoding RNA Cargo Are Emerging as New Modulators for Diabetes Mellitus. Cells (2019) 8(8):853. doi: $10.3390 /$ cells 8080853

66. Bartel DP. Metazoan MicroRNAs. Cell (2018) 173(1):20-51. doi: 10.1016/ j.cell.2018.03.006

67. Pielok A, Marycz K. Non-Coding RNAs as Potential Novel Biomarkers for Early Diagnosis of Hepatic Insulin Resistance. Int J Mol Sci (2020) 21 (11):4182. doi: $10.3390 /$ ijms 21114182

68. Pordzik J, Jakubik D, Jarosz-Popek J, Wicik Z, Eyileten C, De Rosa S, et al. Significance of Circulating microRNAs in Diabetes Mellitus Type 2 and Platelet Reactivity: Bioinformatic Analysis and Review. Cardiovasc Diabetol (2019) 18(1):113. doi: 10.1186/s12933-019-0918-x

69. Eliasson L, Regazzi R. Micro(RNA) Management and Mismanagement of the Islet. J Mol Biol (2020) 432(5):1419-28. doi: 10.1016/j.jmb.2019.09.017

70. Petersen MC, Shulman GI. Mechanisms of Insulin Action and Insulin Resistance. Physiol Rev (2018) 98(4):2133-223. doi: 10.1152/physrev.00063.2017

71. Phua WWT, Wong MXY, Liao Z, Tan NS. An Apparent Functional Consequence in Skeletal Muscle Physiology via Peroxisome ProliferatorActivated Receptors. Int J Mol Sci (2018) 19(5):1425. doi: 10.3390/ ijms19051425

72. Quinn JJ, Chang HY. Unique Features of Long Non-Coding RNA Biogenesis and Function. Nat Rev Genet (2016) 17(1):47-62. doi: 10.1038/nrg.2015.10

73. Zaiou M. circRNAs Signature as Potential Diagnostic and Prognostic Biomarker for Diabetes Mellitus and Related Cardiovascular Complications. Cells (2020) 9(3):659. doi: 10.3390/cells9030659

74. Adela R, Banerjee SK. GDF-15 as a Target and Biomarker for Diabetes and Cardiovascular Diseases: A Translational Prospective. J Diabetes Res (2015) 2015:490842. doi: 10.1155/2015/490842

75. Broedbaek K, Weimann A, Stovgaard ES, Poulsen HE. Urinary 8-Oxo-7,8Dihydro-2'-Deoxyguanosine as a Biomarker in Type 2 Diabetes. Free Radical Biol Med (2011) 51(8):1473-9. doi: 10.1016/j.freeradbiomed.2011.07.007

76. Rathcke CN, Vestergaard H. YKL-40 - An Emerging Biomarker in Cardiovascular Disease and Diabetes. Cardiovasc Diabetol (2009) 8(1):61. doi: $10.1186 / 1475-2840-8-61$
77. Tso AWK, Xu A, Sham PC, Wat NM, Wang Y, Fong CH, et al. Serum Adipocyte Fatty Acid-Binding Protein as a New Biomarker Predicting the Development of Type 2 Diabetes. A 10-Year Prospective Study in a Chinese Cohort. (2007) 30: (10):2667-72. doi: 10.2337/dc07-0413

78. Wang TJ, Ngo D, Psychogios N, Dejam A, Larson MG, Vasan RS, et al. 2Aminoadipic Acid Is a Biomarker for Diabetes Risk. J Clin Invest (2013) 123 (10):4309-17. doi: 10.1172/JCI64801

79. Martinez B, Peplow PV. MicroRNAs in Blood and Cerebrospinal Fluid as Diagnostic Biomarkers of Multiple Sclerosis and to Monitor Disease Progression. Neural Regen Res (2020) 15(4):606-19. doi: 10.4103/16735374.266905

80. Kroh EM, Parkin RK, Mitchell PS, Tewari M. Analysis of Circulating microRNA Biomarkers in Plasma and Serum Using Quantitative Reverse Transcription-PCR (qRT-PCR). Methods (2010) 50(4):298-301. doi: 10.1016/ j.ymeth.2010.01.032

81. Mraz M, Malinova K, Mayer J, Pospisilova S. MicroRNA Isolation and Stability in Stored RNA Samples. Biochem Biophys Res Commun (2009) 390 (1):1-4. doi: 10.1016/j.bbrc.2009.09.061

82. Gloss BS, Dinger ME. The Specificity of Long Noncoding RNA Expression. Biochim Biophys Acta (BBA)-Gene Regul Mech (2016) 1859(1):16-22. doi: 10.1016/j.bbagrm.2015.08.005

83. Qiu G-Z, Tian W, Fu HT, Li CP, Liu B. Long Noncoding RNA-MEG3 Is Involved in Diabetes Mellitus-Related Microvascular Dysfunction. Biochem Biophys Res Commun (2016) 471(1):135-41. doi: 10.1016/j.bbrc.2016.01.164

84. Yan B, Yao J, Liu JY, Li XM, Wang XQ, Li YJ, et al. lncRNA-MIAT Regulates Microvascular Dysfunction by Functioning as a Competing Endogenous RNA. Circ Res (2015) 116(7):1143-56. doi: 10.1161/CIRCRESAHA. 116.305510

Conflict of Interest: The authors declare that the research was conducted in the absence of any commercial or financial relationships that could be construed as a potential conflict of interest.

Publisher's Note: All claims expressed in this article are solely those of the authors and do not necessarily represent those of their affiliated organizations, or those of the publisher, the editors and the reviewers. Any product that may be evaluated in this article, or claim that may be made by its manufacturer, is not guaranteed or endorsed by the publisher.

Copyright (๑) 2021 Chi, Lin, Wang, Zhao, Liao and Wei. This is an open-access article distributed under the terms of the Creative Commons Attribution License (CC BY). The use, distribution or reproduction in other forums is permitted, provided the original author(s) and the copyright owner(s) are credited and that the original publication in this journal is cited, in accordance with accepted academic practice. No use, distribution or reproduction is permitted which does not comply with these terms. 